\title{
Infection Density Dynamics and Phylogeny of Wolbachia Associated with Coconut Hispine Beetle, Brontispa longissima (Gestro) (Coleoptera: Chrysomelidae), by Multilocus Sequence Type (MLST) Genotyping ${ }^{\mathbb{S}}$
}

\author{
Habib Ali ${ }^{1,2}$, Abrar Muhammad ${ }^{1,2}$, and Youming $\mathrm{Hou}^{1,2 *}$ \\ ${ }^{1}$ State Key Laboratory of Ecological Pest Control for Fujian and Taiwan Crops, Fujian Agriculture and Forestry University, Fuzhou 350002, \\ P.R. China \\ ${ }^{2}$ Fujian Provincial Key Laboratory of Insect Ecology, College of Plant Protection, Fujian Agriculture and Forestry University, Fuzhou \\ 350002, P.R. China
}

\begin{abstract}
Received: December 8, 2017
Revised: February 28, 2018

Accepted: March 3, 2018

First published online

April 2, 2018

*Corresponding author

Phone: +86-591-8376-8654;

Fax: +86-591-8376-8654;

E-mail: ymhou@fafu.edu.cn
\end{abstract}

S upplementary data for this paper are available on-line only at http://jmb.or.kr.

pISSN 1017-7825, eISSN 1738-8872

Copyright@ 2018 by

The Korean Society for Microbiology and Biotechnology
The intracellular bacterium Wolbachia pipientis is widespread in arthropods. Recently, possibilities of novel Wolbachia-mediated hosts, their distribution, and natural rate have been anticipated, and the coconut leaf beetle Brontispa longissima (Gestro) (Coleoptera: Chrysomelidae), which has garnered attention as a serious pest of palms, was subjected to this interrogation. By adopting Wolbachia surface protein (wsp) and multilocus sequence type (MLST) genotypic systems, we determined the Wolbachia infection density within host developmental stages, body parts, and tissues, and the results revealed that all the tested samples of B. longissima were infected with the same Wolbachia strain ( $w \mathrm{Log})$, suggesting complete vertical transmission. The MLST profile elucidated two new alleles (ftsZ-234 and coxA-266) that define a new sequence type (ST-483), which indicates the particular genotypic association of B. longissima and Wolbachia. The quantitative real-time PCR analysis revealed a higher infection density in the eggs and adult stage, followed by the abdomen and reproductive tissues, respectively. However, no significant differences were observed in the infection density between sexes. Moreover, the wsp and concatenated MLST alignment analysis of this study with other known Wolbachia-mediated arthropods revealed similar clustering with distinct monophyletic supergroup B. This is the first comprehensive report on the prevalence, infection dynamics, and phylogeny of the Wolbachia endosymbiont in B. longissima, which demonstrated that Wolbachia is ubiquitous across all developmental stages and distributed in the entire body of B. longissima. Understanding the Wolbachia infection dynamics would provide useful insight to build a framework for future investigations, understand its impacts on host physiology, and exploit it as a potential biocontrol agent.

Keywords: Wolbachia, Brontispa longissima, quantitative PCR, MLST, biocontrol agent, symbionthost interactions

\section{Introduction}

Members of the family Chrysomelidae (Insecta: Coleoptera), also known as leaf beetles, are one of the most plentiful, diverse, and successful phytophagous Coleopteran insects, encompassing more than 35,000 species reported worldwide [1]. Owing to its devastating impacts on commercial and ornamental palm cultivation, the pest has garnered a lot of attention [2]. Among the Chrysomelidae family, the genus Brontispa has 22 described species, out of which 17 species have been reported causing considerable damage to various palm species [3]. Brontispa longissima (Gestro) (Coleoptera: Chrysomelidae), commonly known as coconut hispine beetle, is a notorious pest and key defoliator of the 
coconut palm, Cocos nucifera L. (Arecales: Arecaceae) [4]. This pest was originally documented from Papua New Guinea and Indonesia; however, owing to international trade of infested plant materials, the beetle has spread to several other countries, including Tahiti Vanuatu, Samoa, and Southeast and East Asian countries such as the Solomon Islands, Maldives, Thailand, Vietnam, Indonesia, and China, and is rapidly infecting other C. nuciferagrowing regions of the world [5]. Recently, the invasion of $B$. longissima in the newly introduced areas is a matter of great concern and was listed in the Global Invasive-Species Database in 2010. It is also a quarantine pest in China, being first reported on C. nucifera in 2002 from Hainan province (Haiku city) [6]. The dispersal rate of B. longissima is rapid and has already invaded Guangdong, Guangxi, Yunnan, and Fujian provinces $(\approx 30,000$ palm trees are infested), and 17 other cities of China are threatened [6, 7]. It can also severely damage several other palm species, particularly Phoenix canariensis Hortulanorum ex Chabaud [8], Areca catechu L. [9], Washingtonia filifera (Linden ex. Andre) H. Wendland, and Syagrus romanzoffiana (Chamisso) Glassman [10]. They produce 4 to 5 successive generations in a year [11], where the larvae and adults favorably attack the folded fronds of palms, feeding on the tender leaf tissues, which causes severe damage to leaves that results in stunted growth and fruit production and in some cases can cause tree death [4].

Symbiotic associations among the metazoans are prevalent in nature. Insects, being the most diverse, successful, and plenteous animals on Earth have established a robust relationship with microbial symbionts [12]. Insects acquire their symbiotic microflora through maternal transmission (vertical transmission) or environmental transmission (horizontal transmission) [13]. Microbial symbionts play many critical roles in their host's biology and evolution, ranging from food absorption, production of important nutrients [14] such as vitamins, amino acids, and cofactors [15], assistance to adopt new niches [16] or new host plants [17], manipulation of host reproduction (e.g., Cardinium and Wolbachia) $[16,18]$, and protection of the host against natural competitors (parasite, predator, or pathogens) [19]. The composition and infection density of these symbionts vary greatly across the members of the same species, sex, and developmental stages, depending on the physiological conditions of the host and genetics, geographic location, food, and age [20,21]. It is of prime importance to know the infection density of host symbionts to decipher their biological effects and functions. Imperfect vertical transmissions may occur owing to low infection density, whereas high infection frequencies may lead to pathogenesis and therefore negatively impact the fitness of their subject. The host must have evolved some sort of mechanism to control the titer of microbial symbionts and keep them in an appropriate range. One possible mechanism to regulate the infection density is the host and symbiont genotypes. Additionally, symbiont-symbiont interactions can also influence the infection density [22]. The symbionts may be in competition for shared resources, space, or niche and hence regulate their own exploitation to avoid the net damage of the symbiotic system and host fitness [23].

The cytoplasmic inherited bacterium Wolbachia pipientis (Rickettsiales, Rickettsiaceae) is a gram-negative Proteobacteria, naturally inducing dramatic reproductive abnormalities in various hosts through a number of phenotypic behaviors typified as cytoplasmic incompatibility (CI) in various insects, isopods, and mites [24], feminization (F) in isopods and moths [25], killing of male (MK) embryo in weevils, fruit flies, and butterflies [26], and induction of parthenogenesis (IP) in thrips and parasitoid wasps [27]. Generally, Wolbachiainfected hosts are benefited from these phenotypes as they guarantee complete vertical transmission of this symbiont to their offspring. From these above mechanisms, CI is the most exceptional phenotype of Wolbachia. Although the molecular methodology is unknown, CI can be elucidated by a "modification-rescue" scheme, in which Wolbachia "modifies" sperm inside the testes, and the sperm develops immaturely, resulting in demise of the embryo when rightful Wolbachia is not available for the eggs to "rescue" the embryo from the modification [28]. This aspect is critical in biological control; however, the use of Wolbachiainduced CI for pest management is still in its infancy.

The prevalence of Wolbachia infection in arthropod fauna is striking [29] and about $20-80 \%$ of arthropods harbor Wolbachia endosymbionts $[30,31]$. Studies on the genus Wolbachia are mainly focused on phylum Arthropoda owing to the higher prevalence (over 90 arthropods including 5 orders of insects, isopods, spiders, and mites) [32, 33], differences of accessibility, successful transfection in naturally uninfected individuals, and its role in rapid speciation [34]. Phylogenetic studies have indicated that these infectious strains are divided into eight $(\mathrm{A}-\mathrm{H})$ discrete supergroups [35]. Nevertheless, more recent literature has documented some more supergroups designated as A-K super orders [36,37]. Among these taxonomic supergroups, $A$ and $B$ are most likely to be documented in arthropods [38], whereas filarial nematodes, springtails, spiders, and termites are infected by distinct Wolbachia clades (C-D, E, F, $\mathrm{G}$, and $\mathrm{H}$ ) [39-42]. Estimating actual phylogenetic relationships 
for each supergroup will provide valuable insight on the evolution and biology of this symbiont. Although the majority of arthropods are discrete in A and B supergroups, in this study we examined whether Wolbachia from B. longissima $(w \log )$ does actually represent a divergent supergroup A or B on the basis of MLST and Wolbachia wsp genotype sequence. The MLST scheme (gatB, ftsZ, hcpA, $f b p A$, and $\operatorname{cox} A$ ) is advocating the basis to establish accurate characterization of Wolbachia strains and generate phenomenal information about the genetic makup, distributions, and host range of these ubiquitous bacteria [43]. The $w s p$ gene is also a marker to resolve the phylogentic relationship of variable Wolbachia strains [44].

In the present study, we quantified the Wolbachia density dynamics from different developmental stages, body parts, and tissues of $B$. longissima using quantitative real-time PCR (qPCR) assay to normalize with $5.8 \mathrm{~S}$ ribosomal RNA as the housekeeping gene (HkG) for the first time against this beetle. Additionally, by phylogenetic analysis on the basis of MLST and wsp genotyping, we established the phylogenetic relatedness of this interrogation with known Wolbachia-associated arthropod lineages attributed to supergroups A and B to determine the actual position of this fastidious endosymbiont. Here, we attempted to obtain adequate information with the potential to be used for future pest management strategies. Indeed, the genus Wolbachia is opportunistic (parasitic or mutualistic) in nature but needs its status to be confirmed in B. longissima.

\section{Materials and Methods}

\section{Test Specimens}

The specimens used in this report were collected from infested coconut palm trees (Cocos nucifera) from Zhangzhou city $\left(24.5130^{\circ}\right.$ $\mathrm{N}, 117.6471^{\circ} \mathrm{E}$ ), Fujian Province of China and dislodged alive into the laboratory with a natural food source (fresh leaves of $C$. nucifera). The collected population was bred in the laboratory under optimum controlled conditions (temp. $25-28^{\circ} \mathrm{C}, \mathrm{RH} 65-70 \%$, light-dark cycle 12:12) [45] for successive generations as previously described [9]. Specimen identification between males and females of B. longissima (Gestro) was made on the basis of size (male usually smaller than female) and modifications of the last abdominal sternum of the adult, using a stereomicroscope under the supervision of Professor Hou Youming (Fujian Agriculture and Forestry University, China). The experiment was carried out on the F2 or F3 laboratory generations.

\section{DNA Extraction}

Individuals from each mature larvae, 4-6 days old pupae, and 7-9 days old adults (female and male) were randomly selected from F2 or F3 generations, whereas a bunch of newly hatched eggs ( $\approx 50$ eggs) were used for DNA extractions. Prior to DNA extractions, samples (3 individuals/sample) were washed three times with $75 \%$ alcohol and autoclaved double-distilled water. Adult beetles (1-week-old virgin male and female) were dissected for their body parts (head, thorax, and whole abdomen), gut, and reproductive tissues (ovary for female and testes for male) using sterile dissecting equipment, under the microscope, with phosphate buffer saline (PBS) ( $\mathrm{NaCl} 8 \mathrm{~g}, \mathrm{KCl} 0.2 \mathrm{~g}, \mathrm{Na}_{2} \mathrm{HPO}_{4} 1.42 \mathrm{~g}, \mathrm{KH}_{2} \mathrm{PO}_{4}$ $0.27 \mathrm{~g}$, ultrapure water, $\mathrm{pH}$ 7.4). After dissection, samples were carefully transferred to new sterilized $1.5 \mathrm{ml}$ tubes and homogenized in $180 \mu \mathrm{l}$ of ATL (animal tissue lysis) buffer using high-throughput TissueLyser II (Qiagen: Cat No./ID: 85300 at $60 \mathrm{~Hz}$ for $5 \mathrm{~min}$ ) homogenizer. Whole genomic DNA from the samples (eggs, larvae, pupae, and adult females and males) was extracted using a DNeasy Blood and Tissue Kit (Qiagen, USA) following the manufacturer's recommendations with appropriate modifications. Briefly, $20 \mu \mathrm{l}$ of proteinase $\mathrm{K}$ was added to the suspensions and incubated for more than $1 \mathrm{~h}$ at $56^{\circ} \mathrm{C}$. The final elution step was repeated two times in $50 \mu \mathrm{l}$ of $\mathrm{AE}$ buffer. The purity and concentration of the DNA were quantified using a NanoDrop 2000 spectrophotometer (Thermo Scientific, USA) and then run on agarose gel electrophoresis to assess its integrity.

\section{Screening of Wolbachia by Diagnostic PCR}

To interrogate the presence of Wolbachia infection across various developmental stages, body parts, and tissues of B. longissima, diagnostic PCR was conducted with Wolbachia-specific primers targeting specific regions of Wolbachia wsp and MLST genotype (Table 1). Around a 0.6-kb fragment length of $w s p$ and variable length of MLST genes (gatB-369, coxA-402, hcpA-444, ftsZ-435, and $f b p A-423)$ from all the samples were used (Table 1, Figs. S1A and S1B) [44]. Additionally, the $16 \mathrm{~S}$ rRNA bacterial gene sequence with $\approx 1.5 \mathrm{~kb}$ size was also amplified using a universal bacterial primer pair (Table 1 and Fig. S1A) (Sangon Biotech (Shanghai) Co., Ltd., China). Amplification reactions were carried out in a total volume of $25 \mu \mathrm{l}$ that comprised $2 \mu \mathrm{l}$ of template DNA, $12.5 \mu \mathrm{l}$ of $2 \mathrm{X}$ Taq PCR, Master mix (Tiangen Biotechnology Beijing, China), $1 \mu \mathrm{l}$ of each primer $(10 \mu \mathrm{M})$, and $8.5 \mu \mathrm{l}$ of double-distilled water. The thermal cycling profile was $94^{\circ} \mathrm{C}$ for $4 \mathrm{~min}$, followed by 30 cycles of $40 \mathrm{sec}$ at $94^{\circ} \mathrm{C}, 40 \mathrm{sec}$ at $55^{\circ} \mathrm{C}, 1 \mathrm{~min}$ at $72^{\circ} \mathrm{C}$, and a final extension step for $10 \mathrm{~min}$ at $72^{\circ} \mathrm{C}$ for $\operatorname{wsp}(81 \mathrm{~F}-691 \mathrm{R})$, and $94^{\circ} \mathrm{C}$ for $3 \mathrm{~min}, 40 \mathrm{sec}$ at $94^{\circ} \mathrm{C}, 40 \mathrm{sec}$ at $55^{\circ} \mathrm{C}, 1 \mathrm{~min}$ at $72^{\circ} \mathrm{C}$, and a final extension $5 \mathrm{~min}$ at $72^{\circ} \mathrm{C}$ for the $16 \mathrm{~S}$ rRNA gene (27F-1492R). For the MLST genes, PCR protocols available at http://pubmlst.org/ Wolbachia/ [43] with modification of the temperature profiles (gatB and $f b p A$ at $55^{\circ} \mathrm{C}, \operatorname{cox} A$ and $h c p A$ at $50^{\circ} \mathrm{C}$ and $f t s Z$ at $48^{\circ} \mathrm{C}$ ) were used. Furthermore, for the clarification of single or double Wolbachia infection, we used a previously described protocol (https://pubmlst.org/wolbachia/info/amp_seq_double.shtml) (Table S1). MLST analysis was carried out on three DNA samples for each developmental stage, body part, or tissue. 
Table 1. Primer pairs used in this study

\begin{tabular}{|c|c|c|c|c|c|}
\hline Purpose & Primer descriptions & Primer code & Primer Sequence 5'- $3^{\prime}$ & $\begin{array}{l}\text { Fragment } \\
\text { length }(b p)\end{array}$ & Ref. \\
\hline $\begin{array}{l}\text { Universal } \\
\text { bacterial primer }\end{array}$ & $16 \mathrm{~S}$ ribosomal RNA gene & $\begin{array}{l}27 \mathrm{~F} \\
1492 \mathrm{R}\end{array}$ & $\begin{array}{l}\text { AGAGTTTGATCCTGGCTCAG } \\
\text { GGTTACCTTGTTACGACTT }\end{array}$ & $\approx 1,400$ & [9] \\
\hline $\begin{array}{l}\text { Specific for } \\
\text { Wolbachia }\end{array}$ & Wolbachia surface protein & $\begin{array}{l}\text { wsp81F } \\
\text { wsp691R }\end{array}$ & $\begin{array}{l}\text { TGGTCCAATAAGTGATGAAGAAAC } \\
\text { AAAAATTAAACGCTACTCCA }\end{array}$ & $\approx 600$ & [44] \\
\hline \multirow[t]{2}{*}{ qPCR genes } & 5.8S ribosomal RNA & $\begin{array}{l}5.8 S \mathrm{R}-\mathrm{F} \\
5.8 \mathrm{~S} \mathrm{R}-\mathrm{R}\end{array}$ & $\begin{array}{l}\text { AACGGTGGATCACTTGGTTC } \\
\text { ATACGACCCTCAGCCAGGAG }\end{array}$ & $\approx 151$ & This study \\
\hline & Wolbachia surface protein & $\begin{array}{l}\text { wspDi_qF3 } \\
\text { wspDi_qR3 }\end{array}$ & $\begin{array}{l}\text { AGGGCTTTACTCAAAATTGG } \\
\text { CACCAACGTATGGAGTGATAGG }\end{array}$ & $\approx 149$ & [48] \\
\hline \multirow[t]{5}{*}{ MLST genes } & $\begin{array}{l}\text { Glutamyl-tRNA } \\
\text { amidotransferase, subunit B }\end{array}$ & $\begin{array}{l}\text { gatB_F } \\
\text { gatB_R }\end{array}$ & $\begin{array}{l}\text { GAKTTAAAYCGYGCAGGBGTT } \\
\text { TGGYAAYTCRGGYAAAGATGA }\end{array}$ & $\approx 369$ & [43] \\
\hline & Cytochrome $c$ oxidase, subunit I & $\begin{array}{l}\text { COxA_F } \\
\text { COxA_R }\end{array}$ & $\begin{array}{l}\text { TTGGRGCRATYAACTTTATAG } \\
\text { CTAAAGACTTTKACRCCAGT }\end{array}$ & $\approx 402$ & \\
\hline & Conserved hypothetical protein & $\begin{array}{l}\text { hcpA_F } \\
\text { hcpA_R }\end{array}$ & $\begin{array}{l}\text { GAAATARCAGTTGCTGCAAA } \\
\text { GAAAGTYRAGCAAGYTCTG }\end{array}$ & $\approx 444$ & \\
\hline & Cell division protein & $\begin{array}{l}\mathrm{ftsZ} F \\
\mathrm{ftsZ} F\end{array}$ & $\begin{array}{l}\text { ATYATGGARCATATAAARGATAG } \\
\text { TCRAGYAATGGATTRGATAT }\end{array}$ & $\approx 435$ & \\
\hline & Fructose-bisphosphate aldolase & $\begin{array}{l}\text { fbpA_F } \\
\text { fbpA_R }\end{array}$ & $\begin{array}{l}\text { GCTGCTCCRCTTGGYWTGAT } \\
\text { CCRCCAGARAAAAYYACTATTC }\end{array}$ & $\approx 429$ & \\
\hline
\end{tabular}

\section{Cloning and Transformations of $w s p$ and MLST Genes}

PCRs were carried out to amplify the targeted regions of the wsp (Fig. S1) and MLST genes (Table 1). The PCR cycling conditions remained the same as described above. The PCR products, after

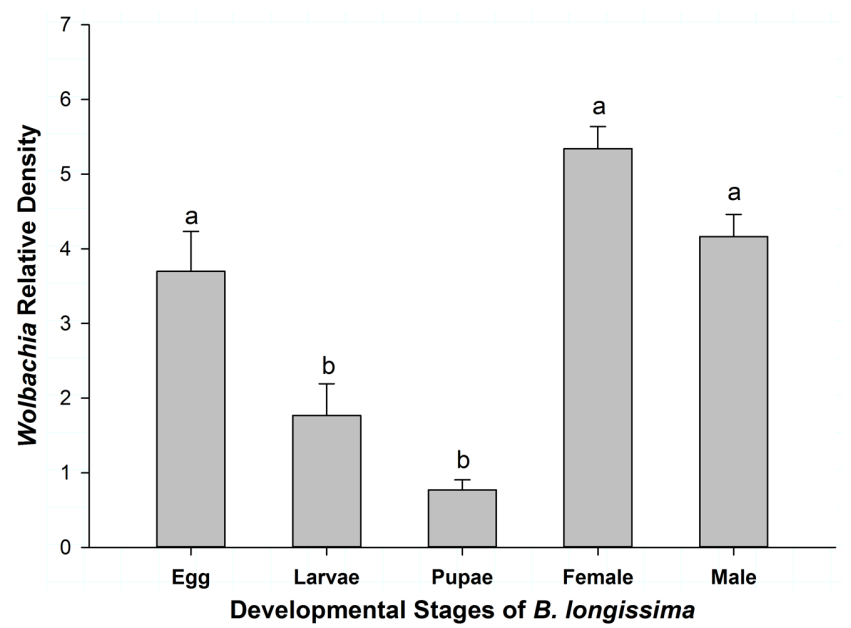

Fig. 1. Variations of Wolbachia density in Brontispa longissima across developmental stages.

The density of Wolbachia is calculated by the ratio between the number of Wolbachia genome relative to that of B. longissima (evaluated by qPCR). At least three biological replicates were run for each developmental stage to quantify the infection density. Tukey's HSD post hoc test was used for multiple comparison at the $p<0.05$ level; the different alphabets indicate the significance level of all tested developmental stages. evaluation for positive amplification verified through gel electrophoresis, were excised and subjected to cloning and transformations. The DNA from the gel was purified with a MiniElute Gel Extraction Kit (Qiagen, USA) and $2 \mu \mathrm{l}$ of DNA was directly ligated into the pGEM T-Easy Cloning Vector (Promega, USA). After ligation, the products were transformed with T1 Competent Cells (Qiagen, USA) following the manufacturer's guidelines. Positive recombinants were sequenced by a commercial sequencing company (BioSune Biotech. Shanghai, China).

\section{Analysis of Sequences Attained from MLST and wsp Genes}

To estimate the origin and closest strains of Wolbachia infections in B. longissima, the homology of all representative sequences $(w s p)$ of this study of Wolbachia was checked using the NCBI BLAST tool (https://blast.ncbi.nlm.nih.gov/Blast.cgi) and their closest sequences were computed (Table S2). Multiple sequence alignment was performed within subjected life stages of B. longissima using the Clustal Omega (http://www.ebi.ac.uk/ Tools/msa/clustalo/) program (Fig. S4). All wsp sequence were analyzed in the wsp database (http://pubmlst.org/wolbachia/ $w s p /$ ) to define the hypervariable regions (HVRs) that are used for Wolbachia strain characterization. Subsequently, for MLST evaluation, five ubiquitous genotype patterns ( gatB, $\operatorname{cox} A, f t s Z$, $f b p A$, and $h c p A$ ) were separately assessed through the data deposited in the Wolbachia MLST database (http://pubmlst.org/ wolbachia/) for the aim to verify the allelic combination that elucidate the sequence types (STs) of Wolbachia mediated with B. longissima. All unwanted sequences from both ends were removed as compared with allelic template provided in the MLST 
database and specified with targeted fragment length.

\section{Assemblage and Phylogenetic Analysis}

Phylogenetic analyses were performed on wsp gene sequences $(\approx 0.6 \mathrm{~kb})$ obtained from different life stages of B. longissima (5 sequences) along with 20 Wolbachia-infected arthropod species [44] from different orders (Table 1) retrieved from GenBank (http://www.ncib.nlm. nih.gov/). Meanwhile, 13 concatenated Wolbachia STs associated with different insect species selected from published data [46] and MLST database used for evaluation with ST-483 isolated from B. longissima were aligned using ClustalW in MEGA 5.05 [47]. After alignment, the sequences were manually corrected when and where necessary to produce an unambiguous multiple sequence alignment. Maximum likelihood (ML) tree topology was computed to develop the phylogenetic relationship. Analysis preference parameters were set and bootstrap analysis was performed with 1,000 pseudo replications. The $\mathrm{CTR}+\mathrm{G}$ model with the lowest Bayesian Information Criterion scores is considered to describe the substitution pattern as the best fit model. Gap spaces were removed manually if necessary.

\section{Measuring the Wolbachia Density Dynamics through qPCR}

qPCR was carried out to measure the relative density of Wolbachia across the tested life stages, body parts, and different tissues of B. longissima. Two forward and reverse primer pairs were designed using Primer3 ver. 0.4.0 software (http:/ / biotools. umassmed.edu/bioapps/primer3_www.cgi) and re-evaluated through Beacon Designer Free Edition - PREMIER Biosoft (http:// www.premierbiosoft.com/qOligo/Oligo.jsp?PID=1). The analysis was conducted on three DNA samples for each stage, body part, or tissue, targeting the Wolbachia wsp gene and normalized with $5.8 \mathrm{~S}$ ribosomal RNA host gene retrieved from the European Nucleotide Archive database (ENA) (http:/ /www.ebi.ac.uk/ena/ data/view/Noncoding:KM186304.1:1232.1416:rRNA\&display=fasta). Fragment lengths of $149 \mathrm{bp}$ for wsp and $151 \mathrm{bp}$ for host $5.8 \mathrm{~S}$ ribosomal RNA genes were amplified in this study, evaluated from the short sequence (185 bp) [48] of B. longissima (KM186304.1) retrieved from ENA by following the above protocols. Prior to qPCR, conventional PCR was carried out for screening the specificity and accuracy of the primer pairs (wspDi_qR3, wspDi_F3 and 5.8S R-F, 5.8SR-R). Amplified PCR products were visualized (1\% electrophoresis gel), excised from the gel (Fig. S2), cloned into the T1 cloning vector, and transformed with T1 Competent Cells (Qiagen, USA). The BLASTn search for the sequenced fragments showed $100 \%$ homology to Wolbachia isolated from Diaphorina citri and B. longissima short sequence (GenBank KF680772.1 and KM186304.1 respectively). After preliminary selection and verification, gene expression through qPCRs $(20 \mu \mathrm{l})$ were conducted in triplicates using the AB 7500 Fast Real-Time PCR System (Applied Biosystems, USA) containing $10 \mu \mathrm{l}$ of $2 \times$ Power SYBR Green Real-Time PCR Master Mix (Applied Biosystems, USA), $1 \mu \mathrm{l}$ of final $5 \mathrm{ng}$ template DNA, and $0.4 \mu \mathrm{l}(5 \mu \mathrm{M})$ of each primer. Amplification conditions consisted of $94^{\circ} \mathrm{C}$ for $30 \mathrm{sec}, 94^{\circ} \mathrm{C}$ for
$5 \mathrm{sec}$, and $60^{\circ} \mathrm{C}$ for $34 \mathrm{sec}$. Primer efficacies were tested through a standard curve $(\approx 90 \mathrm{ng} / \mu \mathrm{l})$ with 5 -fold serial dilutions. The relative Wolbachia density, which is synonymous to the number of Wolbachia per host cell, was estimated on the basis of the ratio of wsp and 5.8S rRNA gene Cq values.

\section{Statistical Analysis}

Wolbachia infection densities among all tested developmental stages, body parts, and different tissues of B. longissima were measure with one-way ANOVA, and differences among average means were compared with Tukey's HSD (honest significant difference) test. Estimation of difference within development stages, body parts, and tissues was evaluated by the independent sample $t$ test. All results were statistically analyzed by SPSS software (ver. 21, SPSS Inc., USA).

\section{Nucleotide Accession Numbers}

All sequences from this study have been deposited to the NCBI GeneBank database under the accession numbers of MG345105 to MG345109 (wsp Accession No.) and MG553908 to MG553932 (MLST Accession No.)

\section{Results}

\section{Validation of Wolbachia Prevalence in the Life Stages,} Body Parts, and Tissues of B. longissima

By mean of the diagnostic PCR approach with wsp gene (wsp-81F, wsp-691R) specific primers, we ascertained Wolbachia infection from all tested developmental stages (i.e., eggs, larvae, pupae, and adult female and male), and designated them as $w \log \mathrm{E}, w \log \mathrm{L}, w \log \mathrm{P}, w \log \mathrm{F}$, and $w \log \mathrm{M}$, respectively (Fig. S1A). Wolbachia infection was also found as positive from the tested body parts and tissues of B. longissima (Fig. S1B). Our initial analysis in this study showed that all subjected samples of B. longissima were fixed $(\approx 0.6 \mathrm{~kb})$ and the fidelity of vertical transmission of this endosymbiont to offsprings was complete, with every specimen being infected (Figs. S1A and S1B). The sequencing results from subjected samples were identical ( $w \log )$, and the un-ambiguous peaks indicated the presence of single Wolbachia strains in any stage. Furthermore, the GenBank homology sequence of every life stage, searched against the NCBI database, showed $>99 \%$ nucleotide BLAST identity with wsp encoded with the European honeybee Apis mellifera capensis (Apidae/Hymenoptera) and Asian citrus psyllid Diaphorina citri (Liviidae/Hemiptera) (Table S2). Multiple sequence alignment with Crustal Omega also showed 99\% identity (Fig. S4). Consequently, these results revealed that a single clad $(w \log )$ of Wolbachia endosymbiont is present across different life stages, with 
Table 2. Infection clads, supergroups, and phenotypic behaviors of Wolbachia associated with various arthropod species based on the $w s p$ gene sequence derived for phylogenetic analysis.

\begin{tabular}{|c|c|c|c|c|c|}
\hline $\begin{array}{c}\text { Arthropod hosts with } \\
\text { Wolbachia-associated strains }\end{array}$ & $\begin{array}{l}\text { Infection } \\
\text { clads }\end{array}$ & $\begin{array}{l}\text { Wolbachia } \\
\text { supergroup }\end{array}$ & $\begin{array}{l}\text { Phenotypic } \\
\text { actions }\end{array}$ & $\begin{array}{l}\text { wsp fragment } \\
\text { length }\end{array}$ & $\begin{array}{c}\text { NCBI } \\
\text { GenBank Acc. No. }\end{array}$ \\
\hline Aedes albopictus wAlbA & Mel & A & $\mathrm{Cl}$ & $655 \mathrm{bp}$ & AF020058.1 \\
\hline Drosophila melanogaster $w \mathrm{Mel}$ & Mel & A & $\mathrm{Cl}$ & $674 \mathrm{bp}$ & AF020065.1 \\
\hline Amblyjoppa fuscipennis $w$ Fus & Fus & A & $\mathrm{T}$ & $589 \mathrm{bp}$ & AF071909.1 \\
\hline Drosophila sechellia $w \mathrm{Ha}$ & $\mathrm{Ha}$ & A & $\mathrm{T}$ & 576 bp & AF020073.1 \\
\hline Glossina morsitans wMors & Mors & A & NK & $564 \mathrm{bp}$ & AF020079.1 \\
\hline Cylindrepomus peregrinus wPer & Mors & A & $\mathrm{T}$ & $564 \mathrm{bp}$ & AF071914.1 \\
\hline Ephestia kuehniella wKue & Kue & A & NK & $561 \mathrm{bp}$ & AF071911.1 \\
\hline Muscidifurax uniraptor wUni & Uni & A & $\mathrm{T}$ & $644 \mathrm{bp}$ & AF020071.1 \\
\hline Apis mellifera capensis wCap-B1 & Cap-B1 & B & $\mathrm{T}$ & $599 \mathrm{bp}$ & AF510085.1 \\
\hline Tribolium confusum wCon & Con & B & $\mathrm{Cl}$ & $555 \mathrm{bp}$ & AF020083 \\
\hline Laodelphax striatellus wStri & Con & B & $\mathrm{Cl}$ & $555 \mathrm{bp}$ & AF020080.1 \\
\hline Apoanagyrus diversicornis wDiv & Div & B & $\mathrm{T}$ & $543 \mathrm{bp}$ & AF071916.1 \\
\hline Trichogramma deion wDei & Dei & B & $\mathrm{T}$ & $555 \mathrm{bp}$ & AF020084.1 \\
\hline Thioalkalimicrobium sibericum wSib & Sib & B & $\mathrm{T}$ & $555 \mathrm{bp}$ & AF071923.1 \\
\hline Trichogramma kaykai wKayB & Kay & B & $\mathrm{T}$ & $555 \mathrm{bp}$ & AF071924.1 \\
\hline Culex pipiens wPip & Pip & B & $\mathrm{Cl}$ & $603 \mathrm{bp}$ & KT964225.1 \\
\hline Diaphorina citri $w \mathrm{Di}$ & $\mathrm{Di}$ & B & NK & $600 \mathrm{bp}$ & KF680772.1 \\
\hline Armadillidium vulgare $w \mathrm{Vul}$ & Vul & B & $\mathrm{F}$ & 596 bp & AF071917.1 \\
\hline Tagosodes orizicolus wOri & Ori & B & $\mathrm{Cl}$ & $552 \mathrm{bp}$ & AF020085.1 \\
\hline Encarsia formosa $w$ For & For & B & $\mathrm{T}$ & $546 \mathrm{bp}$ & AF071918.1 \\
\hline
\end{tabular}

NCBI, National Center for Biotechnology Information; wsp, Wolbachia outer surface protein; NK, not known; T, Thelytoky; MK, Male killing; Cl, cytoplasmic incompatibility; F, Feminization.

widespread distribution in the body parts and tissues of B. longissima. Moreover, MLST genotypic evaluations yielded a new distinct strain, ST-483, which was characterized by explanation of two new alleles (coxA-234 and ftsZ-266), followed by already defined alleles (i.e., gatB-158, fbpA-302, and $h c p A-6)$ assessed on the basis of available data in the Wolbachia MLST database (Table 2). Meanwhile, HVRs analysis of wsp did not show any difference except HVR4, which showed identical HVRs to the database (Table 3), suggesting that this analysis is less sensitive than MLST. Thus, this study clarified Wolbachia infection mediated with B. longissima based on two new alleles and new ST. The same allelic profile and HVRs across the life stages indicates that this bacterium has no or little genetic variability from mother to offsprings.

\section{Wolbachia Infection Dynamics through qPCR Assay}

The qPCR primer pairs (wspDi_qF3 and wspDi_qR3 primers) specific for the Wolbachia wsp gene was recruited as previously described [48], whereas primer pairs of the
HkG (5.8S rRNA) with 149 bp product length (Fig. S3) was developed from the ribosomal gene sequence of $B$. longissima (ENA: sequence KM186304.1). The amplification efficiencies of the linearized plasmids were in line with those of B. longissima DNA samples, with efficiency rates of $\approx 90 \%$ generated for the wsp and $5.8 \mathrm{~S}$ ribosomal RNA genes. The qPCR results indicated that Wolbachia infection densities varied significantly across different developmental stages $\left(F_{4,10}=26.24, p<0.001\right)$ and was higher in eggs (average mean $=3.69$ ) and adults (average mean of female and male, 5.34 and 4.16, respectively) as compared with larvae and pupae as shown in Fig. 1. Furthermore, the infection density within the different host body parts and tissues also varied significantly in both females $\left(F_{4,10}=10.175\right.$, $p=0.001)$ and males $\left(F_{4,10}=8.41, p=0.003\right)$, where the infection density was the highest in the abdomen (average mean $=2.37$ ), followed by reproductive tissues (average mean of female ovary and testes of male, 1.84 and 1.43, respectively), head, thorax, and gut, respectively (Fig. 2). However, in the parallel analysis to compare the infection 
Table 3. Allelic compositions and accession numbers of Wolbachia MLST genotyping (ST-483) and identification of hypervariable regions (HVRs) of the Wolbachia surface protein (WSP) profile from various developmental stages of B. longissima.

\begin{tabular}{|c|c|c|c|c|c|c|c|c|c|c|c|c|c|}
\hline \multirow{2}{*}{$\begin{array}{l}\text { Strain } \\
\text { name }^{a}\end{array}$} & \multirow{2}{*}{$\begin{array}{c}\text { Strain } \\
\mathrm{ID}^{\mathrm{b}}\end{array}$} & \multirow{2}{*}{$\begin{array}{c}w s p \\
\text { (Accession } \\
\text { No.) }\end{array}$} & \multicolumn{4}{|c|}{ WSP profile ${ }^{c}$} & \multirow[b]{2}{*}{$w s p$} & \multicolumn{5}{|c|}{ MLST profile (Accession no.) } & \multirow[b]{2}{*}{$\mathrm{ST}^{\mathrm{d}}$} \\
\hline & & & HVR1 & HVR2 & HVR3 & HVR4 & & gatB & $\operatorname{cox} A$ & hсpA & ftsZ & $f b p A$ & \\
\hline \multirow[t]{2}{*}{$w \log E$} & 1842 & MG345105 & 2 & 142 & 143 & 23 & 720 & (158) & (266) & (6) & (234) & (302) & \multirow[t]{2}{*}{483} \\
\hline & & & & & & & & MG553923 & MG553908 & MG553928 & MG553918 & MG553913 & \\
\hline \multirow[t]{2}{*}{$w \log \mathrm{L}$} & 1843 & MG345106 & 2 & 142 & 143 & 23 & 720 & $(158)$ & (266) & (6) & (234) & (302) & \multirow[t]{2}{*}{483} \\
\hline & & & & & & & & MG553924 & MG553909 & MG553929 & MG553919 & MG553914 & \\
\hline \multirow[t]{2}{*}{$w \log \mathrm{P}$} & 1844 & MG345107 & 2 & 142 & 143 & 23 & 720 & (158) & (266) & (6) & (234) & (302) & \multirow[t]{2}{*}{483} \\
\hline & & & & & & & & MG553925 & MG553910 & MG553930 & MG553920 & MG553915 & \\
\hline \multirow[t]{2}{*}{$w \log F$} & 1845 & MG345108 & 2 & 142 & 143 & 23 & 720 & $(158)$ & (266) & (6) & (234) & (302) & \multirow[t]{2}{*}{483} \\
\hline & & & & & & & & MG553926 & MG553911 & MG553931 & MG553921 & MG553916 & \\
\hline \multirow[t]{2}{*}{$w \log M$} & 1846 & MG345109 & 2 & 142 & 143 & 23 & 720 & (158) & (266) & (6) & (234) & (302) & \multirow[t]{2}{*}{483} \\
\hline & & & & & & & & MG553927 & MG553912 & MG553932 & MG553922 & MG553917 & \\
\hline
\end{tabular}

${ }^{\mathrm{a}}$ Wolbachia strain name assigned according to developmental stages of $B$. longissima ( $w \log \mathrm{E}$ for eggs, $w \operatorname{LogL}$ for $\operatorname{larvae}, w \log \mathrm{P}$ for pupae, $w \log \mathrm{F}$ for female, and $w \log \mathrm{M}$ for male).

${ }^{\mathrm{b}}$ Wolbachia strain identifier given by the MLST database.

'Peptide haplotypes of four consecutive sections of WSP, each section including a hypervariable regions.

${ }^{\mathrm{d}}$ Sequencing type (ST) recognized as a unique allelic profile assigned through the MLST database.

densities between female and male body parts and tissues, the independent sample $t$-test results revealed no significant differences in the regions of head (separate difference $t=0.424, d f=4, p=0.693$ ), thorax (separate difference

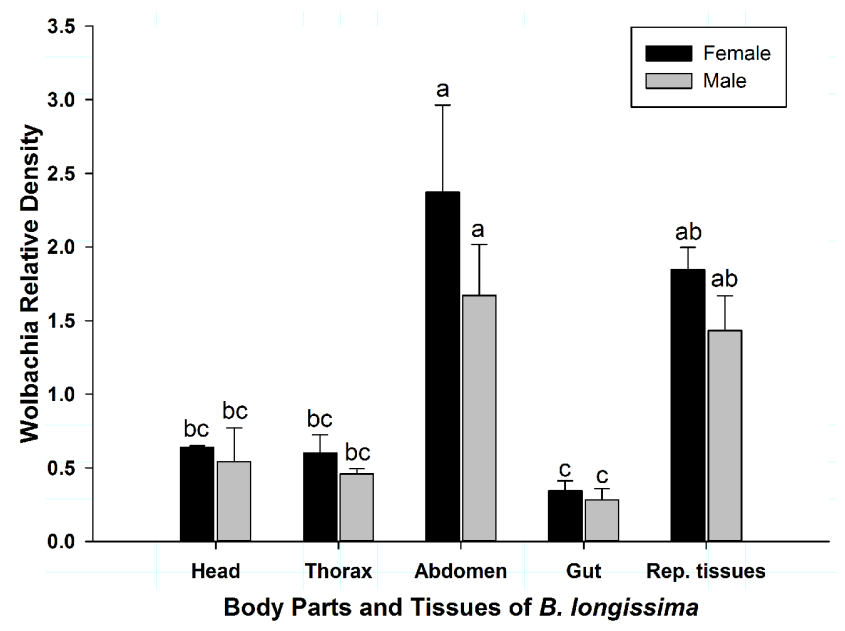

Fig. 2. Variations of Wolbachia density in different body parts and tissues of B. longissima.

The density of Wolbachia is calculated by the ratio between the number of Wolbachia genome relative to that of the B. longissima (evaluated by qPCR). At least three biological replicates were run for each developmental stage to quantify the infection density. Tukey's HSD post hoc test was used for multiple comparison at the $p<0.05$ level; the different alphabets indicate the significance level of all tested body parts. $t=1.092, d f=4, p=0.336$ ), abdomen (separate difference $t=1.022, d f=4, p=0.365$ ), gut (separate difference $t=0.622$, $d f=4, p=0.568$ ), and reproductive tissues (separate difference $t=1.483, d f=4, p=0.212)$. These analyses confirmed that the Wolbachia infection density in this beetle (B. longissima) was influenced by the host developmental stages and body parts or tissues, but not by the gender (male and female).

\section{Phylogenetic Analysis and Group Interference}

The supergroup A or B specific primers (Table S1) inferred the single infection of Wolbachia supergroup B in B. longissima. To evaluate the phylogenetic relationship between the Wolbachia strains isolated from B. longissima across various developmental stages, we constructed phylogenetic trees (Figs. 3A and 3B) of a total of 20 arthropod nucleotide sequences using the ML program (Table 1), and the tree topology of all subjected sequences ( $\approx 0.6 \mathrm{~kb}$ ) confirmed the partition of Wolbachia entailments into two supergroups (A and B). The wsp gene sequence of this study showed monophyletic similarity to that of Wolbachia in the sister group from A. m. capensis (Apidae: Hymenoptera) and D. citri (Kuwayama) (Psyllidae: Hemiptera) and attributed to lineages from Wolbachia (Con and Di subgroup) $w$ Cap-B1 and $w \mathrm{Di}$ strains, which were conclusively classified into supergroup B (Fig. 3A). Similarly, the concatenated MLST sequence (2,073 bp) from B. longissima and those of Wolbachia infecting other organisms from different supergroups $(\mathrm{A}, \mathrm{B}, \mathrm{D}, \mathrm{F}$, and $\mathrm{H})$ were aligned 

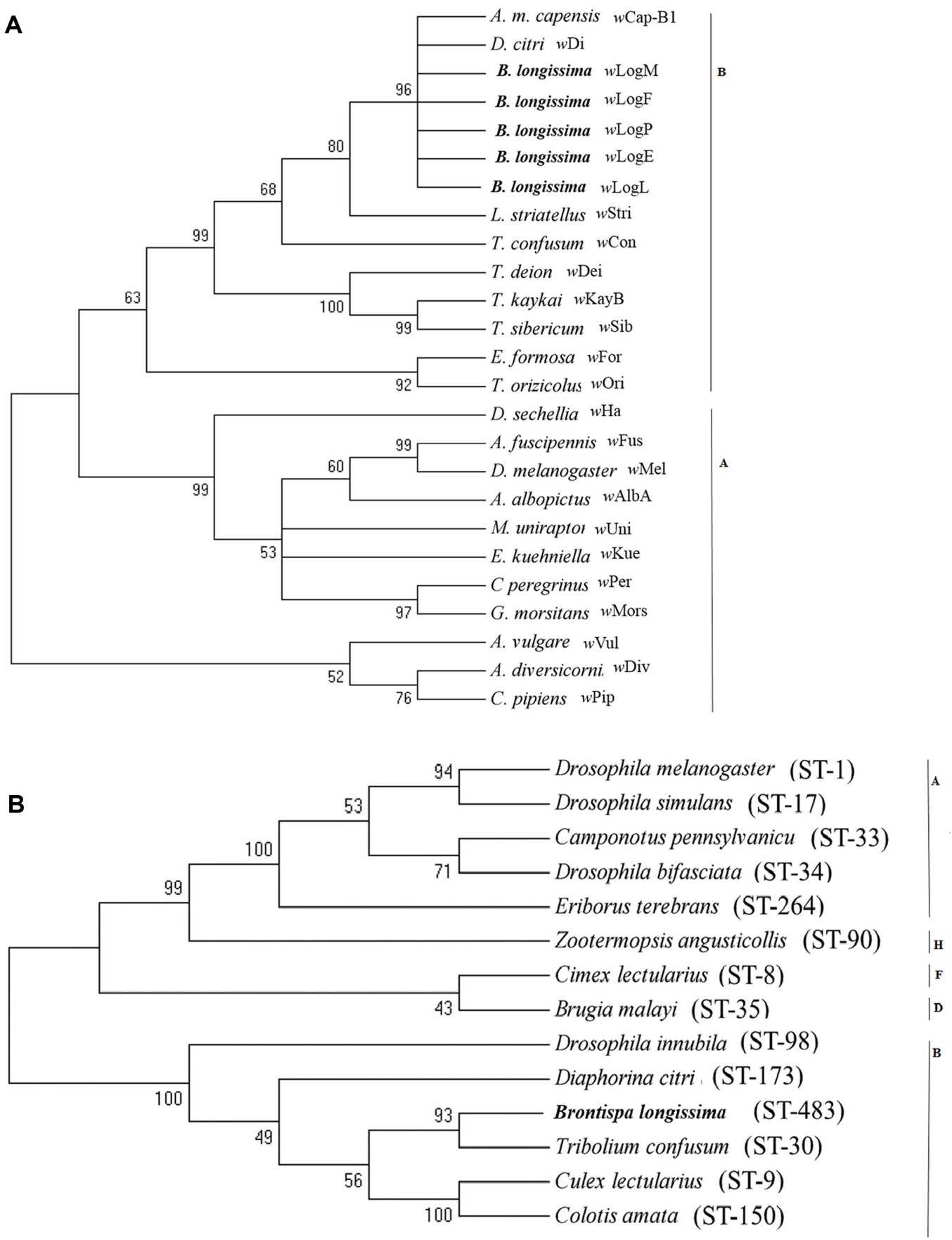

Fig. 3. Phylogenetic placement of Wolbachia strains from B. longissima ( $w \log E$ for eggs, $w \log L$ for larvae, $w \log P$ for pupae, $w \log F$ for female, and $w \log \mathrm{M}$ for male) (bold) with known Wolbachia symbionts from various arthropods, as constructed by the maximum likelihood (ML) program using MEGA (ver. 5.05).

(A) ML phylogenetic tree of the wsp gene $(\approx 600 \mathrm{bp})$ with ( 20 nucleotide sequence) $8 / 20$ and $12 / 20$ sequence of supergroup A and B, respectively, from various arthropods assembled and aligned together for phylogenetic analysis. Nomenclature of Wolbachia strains and groups are according to their names of host species from which they were identified. The letters A and B indicate the Wolbachia supergroups. (B) ML phylogenetic tree of concatenated MLST data (2,073 or 2,079 bp). Phylogeny showing relatedness of B. longissima (ST-483) (bold) with Wolbachia-mediated arthropods (a total of 13 sequence) 5/13,5/13,1/13,1/13, and 1/13 nucleotide sequence belonging to Wolbachia supergroups $\mathrm{A}, \mathrm{B}, \mathrm{H}, \mathrm{F}$, and $\mathrm{D}$, respectively. Alphabet letters (A, B, H, F, and D) indicate different Wolbachia supergroups. All Wolbachia MLST strains were retrieved from the MLST database (http://pubmlst. org/ wolbachia/). 
together, and ML analysis revealed higher monophyletic relatedness to confused flour beetle, Tribolium confusum (Jacquelin du Val) (Tenebrionidae: Coleoptera) belonging to the same supergroup B (Fig. 3B). Considering the homology in Wolbachia sequences across different developmental stages, only one MLST locus was used in strain-based ML chromatogram evaluation analysis. Therefore, on the basis of both phylogenetic analysis to Wolbachia infecting $B$. longissima individuals with other arthropods, the incidence of Wolbachia in B. longissima belongs to supergroup B.

\section{Discussions}

Wolbachia infection is widespread among the natural populations of various insects. Detection of Wolbachia using diagnostic PCR-based approach targeting the wsp gene regions confirmed the Wolbachia infection from various developmental stages (Fig. S1A), body parts, and reproductive tissue (Fig. S1B) of $B$. longissima, which are the novel findings reported here. The wsp gene is commonly used as a strain typing marker [49] and taxonomical sequence tool for micro- and macro classification, as well as identification of new lineages of the genus Wolbachia [41, 50]. Previously, Wolbachia infection and classification using diagnostic PCR assays have been reported by Zhou et al. [44]. The ubiquitous presence of Wolbachia infection in our study is analogous with the interpretation of cytoplasmicinherited bacteria, mainly transfers from mother to offsprings but may also have undergone broadly intertaxon transmission even within or between different orders of insects. These cytoplasmic endosymbionts presumably conflict the expressions and elicit reproductive modification of various insect communities through a number of phenotypic behaviors, such as cytoplasmic incompatibility, parthenogenesis, feminization, and male killing. Nonetheless, phenotypic expressions (cytoplasmic incompatibility, parthenogenesis, feminization, male killing, manipulating effects, or resistance against pathogens) [51] of Wolbachia are the possibilities having no report from B. longissima, and therefore, further studies are needed to investigate these biological roles.

Here, we have provided the first comprehensive quantification analysis of Wolbachia infection densities across different life stages, body parts, and tissues of B. longissima by qPCR, normalized with a component of the large ribosomal subunit, 5.8S rRNA, as the endogenous control gene. Quantitative PCR is commonly employed in gene expression analysis. Nevertheless, assortment of suitable HkGs for normalization is crucial to achieve accurate and reliable results. Published literature has indicated that the 5.8S rRNA gene representes a uniform expression, more stable (in contrast with 6 commonly used reference genes: $\beta$-2-microglobolin (B2M), glyceraldehyde3-phosphate dehydrogenase (GAPDH), $\beta$-glucuronidase (GUSB), peptidylprolyl isomerase A (PPIA), phosphoglycerate kinase 1 (PGK1), and TATA-box binding protein (TBP) in collapsed lung tissues of neonatal somatic cell nuclear transfer-derived cattle), conserved, and broadly used as a HkG [52]. A similar study was conducted to compare the 5.8S rRNA gene in the carmine spider mite, Tetranychus cinnabarinus (Boisduval) (Acarina: Tetranychidae), with other commonly used HkGs and validated its stability of expression under specific experimental conditions [53]. Another study rectified the stability evaluated from miRNAs and non-coding small RNAs [54] and thus showed uniformity among all cell types and experimental systems. Furthermore, the linearized plasmids of wsp and 5.8S rRNA and genomic DNA of B. longissima were amplified within the acceptable range of efficiency [55].

Numerous studies have put forward explanations for the observed variations in the infection density of Wolbachia across diverse taxonomic categories or ecological guilds [31]. In the present study, detection of Wolbachia using qPCR approaches suggested that the endosymbiont is present in all observed samples of B. longissima, although there is evident variations across developmental life stages and was the highest in the eggs and adults as compared with others. In line with this, a recent study to measure the Wolbachia infection dynamics has reported the highest Wolbachia density in the eggs and adult stage as compared with the larvae and pupae, which suggested that the percentage of Wolbachia density increases with the proceeding of developmental stage [56]. In the study of D. citri endosymbionts, a similar trend of positive correlation was observed between the growth pattern of symbionts and host development [57]. The fluctuations in the Wolbachia infection within the adult stage might have been due to the relative size variation of host tissues. This suggests that Wolbachia evolutionary consequences change with proceeding developmental stages and ultimately should exhibit the complex Wolbachia-host interactions. Additionally, the higher infection density in the abdomen and reproductive tissues of female as compared with male adults in B. longissima indicates that Wolbachia preferably colonized the abdomen and reproductive tissues of females, consistent with similar observations reported in fruit fly Drosophila simulans [58], two plant-hoppers species, Laodelphase striatellus and Sogatella furcifera [59], and Aedes 
albopictus [60]. Moreover, the higher infection frequencies of Wolbachia in females also may be consequently higher than males due to a much greater size of the ovaries relative to the testes, while overall high frequencies in the abdomen and reproductive tissues compared with other body parts and tissues from both adults are a reason to improve successive transmission of the bacterium to next generations. Wolbachia is mostly present in somatic and germline tissue [60] and vertically transmitted into various hosts to modify their reproduction; hence, it has been supposed that they just infect the reproductive tissues of the host. Conflicting with this assumption, we showed that Wolbachia is abundantly distributed in the developmental stages (Fig. S1A) and whole body, including head, thorax, abdomen, and gut and reproductive tissues (Fig. S1B), which explains their wide distribution within invertebrate animals. In fact, the reason for the high abundance within germline cells and efficient transmission to the various insect species is just because of the usage of this bacterium as a spindle apparatus during cell partitioning [61] and effective travel to the kinesin and dynein motors within the host cells [62]. The findings of Wolbachia in B. longissima are also similar to previous studies on the prevalence of Wolbachia in various insects $[63,64]$.

In the recent few years, most of the research focus has increased regarding Wolbachia infection in weevils [65] and more than 40 weevil species are reported for Wolbachia infection, which is arguably a larger number than from any other insect group [37, 66]. This is contrary to the assumption that closely related species should trade off Wolbachia strains repeatedly and thus are expected to be associated with similar strains. A broad majority of similar Wolbachia strains have been identified in weevils with unknown ecological interactions. The maximum intimate associations of B. longissima via wsp analysis were found from Wolbachia strains in A. m. carpensis and D. citri (Kuwayama) and T. confusum (Jacquelin du Val) through concatenated MLST analysis, which clearly indicates the horizontal transmission of Wolbachia infections evaluated through ML analysis. All discovered Wolbachia strains in weevils belong to supergroup A or B, except Rhinocyllus conicus (Curculionidae: Coleoptera) that is infected by a strain from supergroup $\mathrm{F}$, which is usually found in true bugs, termites, and nematodes [50,67]. A study conducted to investigate the Wolbachia infection status from 40 weevil species from central Europe rectified that around 15\% weevils infected with Wolbachia belong to supergroup A, $10 \%$ belong to supergroup $\mathrm{B}$, and $15 \%$ belong to both $\mathrm{A}$ and $\mathrm{B}$, while the remaining $60 \%$ were uninfected [68]. Similarly, our phylogenetic analysis from both datasets of endosymbiotic Wolbachia in B. longissima clearly indicates the monophyletic supergroup B (Figs. 3A and 3B). Thus, irrespective of that case (F supergroup), most of the weevil species infections are classified into supergroups A and B. The apparent discrepancies in these analyses may lead to the variable impact of Wolbachia with host resulted from different evolutionary consequences, which reflect complex Wolbachia-host interactions but yet need to be determined in B. longissima.

Evidence is mounting on the various phenotypic behaviors of Wolbachia (CI, IP, F, and MK) inducing dramatic reproductive abnormalities that greatly influence the population demographics of host insect species [24, 27], which makes it a potentially useful tool for efficient pest management [69, 70]. Various effects from Wolbachia induced CI may contribute to pest control. Analogous to the sterile insect technique (SIT), Wolbachia-infected males in biological control programs when released in the field may suppress the pest population by infecting them with CI-inducing Wolbachia, which decreases host fecundity [69, 71]. Indeed, a recent literature rectified that Wolbachiatransfected mosquitoes, Aedes aegypti, were used successfully to block dengue transmission in Australia with no adverse effect on host physiology [72]. In addition, Wolbachia can be used as a potential tool for the introduction of favorable genes into insect population that can suppress the pathogen transmission by insect vectors, such as mosquito (genes that reduce the transmission of human pathogens) [72] and plant-hoppers [73]. In different host species, the Wolbachia-induced CI can vary from $0 \%-100 \%$ [74]. In broad spectrum, the palm pests (B. longissima) contaminate foods (coconut oil, date palm, etc.) and therefore, potential effects of Wolbachia on human fitness may require consideration. Until now, nothing was known about the effects of oral administration of Wolbachia endosymbionts on human health. However, some Wolbachia strains that are indispensable endosymbionts of the pathogenic nematodes causing lymphatic filariasis and river blindness induce severe inflammatory problems in humans when they are released into the blood $[75,76]$. In this case, the present study on Wolbachia prevalence and quantification will help us unravel the nature of this symbiont-host interaction and propose the future prospects of Wolbachia-mediated insect pest management strategies. However, further studies are needed to explore the capacities of the Wolbachia-arthropod relationships and their implementation against vector and disease management. In conclusion, this study is the first report of the prevalence, infection status, and phylogeny of 
Wolbachia endosymbionts in B. longissima and demonstrated that Wolbachia is ubiquitous across all developmental stages and distributed in the entire body of B. longissima, which provides a roadmap to find out its biological impacts and symbiont host associations.

\section{Acknowledgments}

We are very grateful for the grants from the National Key R \& D Program of China (2017YFC1200605) and Fujian Science and Technology Special Project (2017NZ0003-1-6).

\section{Conflict of Interest}

The authors have no financial conflicts of interest to declare.

\section{References}

1. Jolivet P, Santiago-Blay JA, Schmitt M. (eds.). 2008. Research on Chrysomelidae. Brill Leiden, The Netherlands.

2. Wesseler J, Fall EH. 2010. Potential damage costs of Diabrotica virgifera virgifera infestation in Europe - the 'no control' scenario. J. Appl. Entomol. 134: 385-394.

3. Staines C. 2012. Catalog of the hispines of the World (Coleoptera: Chrysomelidae: Cassidinae). Tribe Cryptonychini. Online publication available from http://entomology.si.edu/ Collections_Coleoptera-Hispines.html (last accessed January 2014).

4. Konishi K, Nakamura S, Takasu K. 2007. Invasion of the coconut hispine beetle, Brontispa longissima: current situation and control measures in Asia. Presented at the NIAES International Symposium 2007. Invasive Alien Species in Monsoon Asia: Status and Control. Epochal Tsukuba, Japan, October 22-23, 2007.

5. Sugeno W, Kawazu K, Takano S, Nakamura S, Mochizuki A. 2011. Suitability of monocots for rearing alien coconut pest Brontispa longissima (Coleoptera: Chrysomelidae). Ann. Entomol. Soc. Am. 104: 682-687.

6. Lu Y, Zeng L, Wang L, Zhou R. 2003. Risk analysis of palm leaf beetle Brontispa longissima (Gestro). Entomol. J. East Chin. 13: $17-20$.

7. Zhang X, Tang B, Hou Y. 2015. A rapid diagnostic technique to discriminate between two pests of palms, Brontispa longissima and Octodonta nipae (Coleoptera: Chrysomelidae), for quarantine applications. J. Econ. Entomol. 108: 95-99.

8. Yamashita A, Takasu K. 2010. Suitability of potential host plants in Japan for immature development of the coconut hispine beetle, Brontispa longissima (Gestro) (Coleoptera: Chrysomelidae). Japan Agric. Res. Q. 44: 143-149.

9. Hou Y, Miao Y, Zhang Z. 2014. Study on life parameters of the invasive species Octodonta nipae (Coleoptera: Chrysomelidae) on different palm species, under laboratory conditions. J. Econ. Entomol. 107: 1486-1495.

10. Wu Q, Zeng L, Sun J-C, Liang G, Lu Y. 2006. Control efficiency of Metarhizium anisopliae on Brontispal longissima (Gestro) in field. J. Shandong Agric. Univ. 37: 568.

11. Zhong M, Shen Z-R. 2004. Infection of the endosymbiont Wolbachia in population of Trichogramma evanescens in China. Acta Entomol. Sin. 6: 732-737.

12. Harris HL, Brennan LJ, Keddie BA, Braig HR. 2010. Bacterial symbionts in insects: balancing life and death. Symbiosis 51: 37-53.

13. Mason CJ, Raffa KF. 2014. Acquisition and structuring of midgut bacterial communities in gypsy moth (Lepidoptera: Erebidae) larvae. Environ. Entomol. 43: 595-604.

14. Dillon R, Dillon V. 2004. The gut bacteria of insects: nonpathogenic interactions. Annu. Rev. Entomol. 49: 71-92.

15. Douglas AE. 2009. The microbial dimension in insect nutritional ecology. Funct. Ecol. 23: 38-47.

16. Moya A, Peretó J, Gil R, Latorre A. 2008. Learning how to live together: genomic insights into prokaryote-animal symbioses. Nat. Rev. Genet. 9: 218-229.

17. Hosokawa T, Kikuchi Y, Nikoh N, Shimada M, Fukatsu T. 2006. Strict host-symbiont cospeciation and reductive genome evolution in insect gut bacteria. PLoS Biol. 4: e337.

18. Yen JH, Barr AR. 1973. The etiological agent of cytoplasmic incompatibility in Culex pipiens. J. Invertebr. Pathol. 22: 242-250.

19. Jaenike J, Unckless R, Cockburn SN, Boelio LM, Perlman SJ. 2010. Adaptation via symbiosis: recent spread of a Drosophila defensive symbiont. Science 329: 212-215.

20. Lu F, Kang X, Lorenz G, Espino L, Jiang M, Way MO. 2014. Culture-independent analysis of bacterial communities in the gut of rice water weevil (Coleoptera: Curculionidae). Ann. Entomol. Soc. Am. 107: 592-600.

21. Pernice M, Simpson SJ, Ponton F. 2014. Towards an integrated understanding of gut microbiota using insects as model systems. J. Insect Physiol. 69: 12-18.

22. Mouton L, Henri H, Bouletreau M, Vavre F. 2003. Strainspecific regulation of intracellular Wolbachia density in multiply infected insects. Mol. Ecol. 12: 3459-3465.

23. Koga R, Tsuchida T, Fukatsu T. 2003. Changing partners in an obligate symbiosis: a facultative endosymbiont can compensate for loss of the essential endosymbiont Buchnera in an aphid. Proc. R. Soc. Lond. B 270: 2543-2550.

24. Hoffmann A, Turelli M. 1997. Cytoplasmic incompatibility in insects, pp. 42-80. In O'Neill SL, Werren JH, Hoffmann AA (eds.). Influential Passengers. Oxford University Press, New York.

25. Bouchon D, Rigaud T, Juchault P. 1998. Evidence for widespread Wolbachia infection in isopod crustaceans: molecular identification and host feminization. Proc. R. Soc. Lond. B 265: 1081-1090.

26. Fialho RF, Stevens L. 2000. Male-killing Wolbachia in a flour 
beetle. Proc. R. Soc. Lond. B 267: 1469-1473.

27. Pannebakker BA, Pijnacker LP, Zwaan BJ, Beukeboom LW. 2004. Cytology of Wolbachia-induced parthenogenesis in Leptopilina clavipes (Hymenoptera: Figitidae). Genome 47: 299303.

28. Werren JH. 1997. Biology of Wolbachia. Annu. Rev. Entomol. 42: 587-609.

29. Jiggins FM, Bentley JK, Majerus ME, Hurst GD. 2001. How many species are infected with Wolbachia? Cryptic sex ratio distorters revealed to be common by intensive sampling. Proc. R. Soc. Lond. B 268: 1123-1126.

30. Jeyaprakash A, Hoy M. 2000. Long PCR improves Wolbachia DNA amplification: wsp sequences found in $76 \%$ of sixtythree arthropod species. Insect Mol. Biol. 9: 393-405.

31. Werren JH, Windsor DM. 2000. Wolbachia infection frequencies in insects: evidence of a global equilibrium? Proc. $R$. Soc. Lond. B 267: 1277-1285.

32. Werren JH, Windsor D, Guo L. 1995. Distribution of Wolbachia among neotropical arthropods. Proc. R. Soc. Lond. B 262: 197-204.

33. Oh HW, Kim MG, Shin SW, Bae KS, Ahn YJ, Park HY. 2000. Ultrastructural and molecular identification of a Wolbachia endosymbiont in a spider, Nephila clavata. Insect Mol. Biol. 9: 539-543.

34. Tsuchida T, Koga R, Fukatsu T. 2004. Host plant specialization governed by facultative symbiont. Science 303: 1989.

35. Bordenstein SR, Paraskevopoulos C, Hotopp JCD, Sapountzis P, Lo N, Bandi C, et al. 2009. Parasitism and mutualism in Wolbachia: what the phylogenomic trees can and cannot say. Mol. Biol. Evol. 26: 231-241.

36. Salunke BK, Salunkhe RC, Patole MS, Shouche YS. 2010. Wolbachia and termite association: present status and future implications. J. Biosci. 35: 171-175.

37. Ali H, Hou Y, Tang B, Shi ZH, Huang B, Muhammad A, et al. 2016. A way of reproductive manipulation and biology of Wolbachia pipientis. J. Exp. Biol. Agric. Sci. 4: 156-168.

38. Werren JH, Zhang W, Guo LR. 1995. Evolution and phylogeny of Wolbachia: reproductive parasites of arthropods. Proc. R. Soc. Lond. B 261: 55-63.

39. Vandekerckhove TT, Watteyne S, Willems A, Swings JG, Mertens J, Gillis M. 1999. Phylogenetic analysis of the $16 \mathrm{~S}$ rDNA of the cytoplasmic bacterium Wolbachia from the novel host Folsomia candida (Hexapoda, Collembola) and its implications for wolbachial taxonomy. FEMS Microbiol. Lett. 180: 279-286.

40. Rowley SM, Raven RJ, McGraw EA. 2004. Wolbachia pipientis in Australian spiders. Curr. Microbiol. 49: 208-214.

41. Casiraghi M, Bordenstein S, Baldo L, Lo N, Beninati T, Wernegreen J, et al. 2005. Phylogeny of Wolbachia pipientis based on gltA, groEL and ftsZ gene sequences: clustering of arthropod and nematode symbionts in the F supergroup, and evidence for further diversity in the Wolbachia tree. Microbiology 151: 4015-4022.
42. Lo N, Paraskevopoulos C, Bourtzis K, O'Neill S, Werren J, Bordenstein S, et al. 2007. Taxonomic status of the intracellular bacterium Wolbachia pipientis. Int. J. Syst. Evol. Microbiol. 57: 654-657.

43. Baldo L, Hotopp JCD, Jolley KA, Bordenstein SR, Biber SA, Choudhury RR, et al. 2006. Multilocus sequence typing system for the endosymbiont Wolbachia pipientis. Appl. Environ. Microbiol. 72: 7098-7110.

44. Zhou W, Rousset F, O'Neill S. 1998. Phylogeny and PCRbased classification of Wolbachia strains using wsp gene sequences. Proc. R. Soc. Lond. B 265: 509-515.

45. Kaakeh W. 2005. Longevity, fecundity, and fertility of the red palm weevil, Rynchophorus ferrugineus Olivier (Coleoptera: Curculionidae) on natural and artificial diets. Emirates $J$. Agric. Sci. 17: 23-33.

46. Avtzis DN, Doudoumis V, Bourtzis K. 2014. Wolbachia infections and mitochondrial diversity of two chestnut feeding Cydia species. PLoS One 9: e112795.

47. Tamura K, Peterson D, Peterson N, Stecher G, Nei M, Kumar S. 2011. MEGA5: molecular evolutionary genetics analysis using maximum likelihood, evolutionary distance, and maximum parsimony methods. Mol. Biol. Evol. 28: 2731-2739.

48. Hoffmann M, Coy M, Pelz-Stelinski K. 2014. Wolbachia infection density in populations of the Asian citrus psyllid (Hemiptera: Liviidae). Environ. Entomol. 43: 1215-1222.

49. Kyei-Poku G, Colwell D, Coghlin P, Benkel B, Floate K. 2005. On the ubiquity and phylogeny of Wolbachia in lice. Mol. Ecol. 14: 285-294.

50. Lo N, Casiraghi M, Salati E, Bazzocchi C, Bandi C. 2002. How many Wolbachia supergroups exist? Mol. Biol. Evol. 19: 341-346.

51. Hamm CA, Begun DJ, Vo A, Smith CC, Saelao P, Shaver AO, et al. 2014. Wolbachia do not live by reproductive manipulation alone: infection polymorphism in Drosophila suzukii and D. subpulchrella. Mol. Ecol. 23: 4871-4885.

52. Liu Y, Zhang Y, Jiang Q, Rao M, Sheng Z, Zhang Y, et al. 2015. Identification of valid housekeeping genes for realtime quantitative PCR analysis of collapsed lung tissues of neonatal somatic cell nuclear transfer-derived cattle. Cell. Reprogram. 17: 360-367.

53. Sun W, Jin Y, He L, Lu W-C, Li M. 2010. Suitable reference gene selection for different strains and developmental stages of the carmine spider mite, Tetranychus cinnabarinus, using quantitative real-time PCR. J. Insect Sci. 10: 208.

54. Song H, Zhang X, Shi C, Wang S, Wu A, Wei C. 2016. Selection and verification of candidate reference genes for mature microRNA expression by quantitative RT-PCR in the tea plant (Camellia sinensis). Genes 7: 25.

55. Bustin SA. 2000. Absolute quantification of mRNA using real-time reverse transcription polymerase chain reaction assays. J. Mol. Endocrinol. 25: 169-193.

56. Ming Q-L, Shen J-F, Cheng C, Liu C-M, Feng Z-J. 2015. Wolbachia infection dynamics in Tribolium confusum (Coleoptera: 
Tenebrionidae) and their effects on host mating behavior and reproduction. J. Econ. Entomol. 108: 1408-1415.

57. Dossi FCA, da Silva EP, Cônsoli FL. 2014. Population dynamics and growth rates of endosymbionts during Diaphorina citri (Hemiptera, Liviidae) ontogeny. Microb. Ecol. 68: 881-889.

58. Rousset F, Braig HR, O'Neill SL. 1999. A stable triple Wolbachia infection in Drosophila with nearly additive incompatibility effects. Heredity 82: 620-627.

59. Noda H, Koizumi Y, Zhang Q, Deng K. 2001. Infection density of Wolbachia and incompatibility level in two planthopper species, Laodelphax striatellus and Sogatella furcifera. Insect Biochem. Mol. Biol. 31: 727-737.

60. Dobson SL, Bourtzis K, Braig HR, Jones BF, Zhou W, Rousset F, et al. 1999. Wolbachia infections are distributed throughout insect somatic and germ line tissues. Insect Biochem. Mol. Biol. 29: 153-160.

61. Kose H, Karr TL. 1995. Organization of Wolbachia pipientis in the Drosophila fertilized egg and embryo revealed by an anti-Wolbachia monoclonal antibody. Mech. Dev. 51: 275-288.

62. Serbus LR, Sullivan W. 2007. A cellular basis for Wolbachia recruitment to the host germline. PLoS Pathog. 3: e190.

63. Kondo N, Shimada M, Fukatsu T. 1999. High prevalence of Wolbachia in the azuki bean beetle Callosobruchus chinensis (Coleoptera, Bruchidae). Zool. Sci. 16: 955-962.

64. Cheng Q, Ruel T, Zhou W, Moloo S, Majiwa P, O'Neill S, et al. 2000. Tissue distribution and prevalence of Wolbachia infections in tsetse flies, Glossina spp. Med. Vet. Entomol. 14: 44-50.

65. Rożek M, Lachowska D, Holecovà M, Kajtoch Ł. 2009. Karyology of parthenogenetic weevils (Coleoptera, Curculionidae): do meiotic prophase stages occur? Micron 40: 881-885.

66. Toju H, Fukatsu T. 2011. Diversity and infection prevalence of endosymbionts in natural populations of the chestnut weevil: relevance of local climate and host plants. Mol. Ecol. 20: 853-868.

67. Rasgon JL, Scott TW. 2004. Phylogenetic characterization of
Wolbachia symbionts infecting Cimex lectularius L. and Oeciacus vicarius Horvath (Hemiptera: Cimicidae). J. Med. Entomol. 41: 1175-1178.

68. Lachowska D, Kajtoch $€$, Knutelski S. 2010. Occurrence of Wolbachia in central European weevils: correlations with host systematics, ecology, and biology. Entomol. Exp. Appl. 135: 105-118.

69. Zabalou S, Riegler M, Theodorakopoulou M, Stauffer C, Savakis C, Bourtzis K. 2004. Wolbachia-induced cytoplasmic incompatibility as a means for insect pest population control. Proc. Natl. Acad. Sci. USA 101: 15042-15045.

70. Bourtzis K. 2008. Wolbachia-based technologies for insect pest population control. Adv. Exp. Med. Biol. 627: 104-113.

71. Calvitti M, Moretti R, Lampazzi E, Bellini R, Dobson SL. 2010. Characterization of a new Aedes albopictus (Diptera: Culicidae)-Wolbachia pipientis (Rickettsiales: Rickettsiaceae) symbiotic association generated by artificial transfer of the wPip strain from Culex pipiens (Diptera: Culicidae). J. Med. Entomol. 47: 179-187.

72. Walker T, Johnson P, Moreira L, Iturbe-Ormaetxe I, Frentiu F, McMeniman C, et al. 2011. The wMel Wolbachia strain blocks dengue and invades caged Aedes aegypti populations. Nature 476: 450-453.

73. Hoffmann AA, Ross PA, Rašić G. 2015. Wolbachia strains for disease control: ecological and evolutionary considerations. Evol. Appl. 8: 751-768.

74. Merçot H, Charlat S. 2004. Wolbachia infections in Drosophila melanogaster and D. simulans: polymorphism and levels of cytoplasmic incompatibility. Genetica 120: 51-59.

75. Cross HF, Haarbrink M, Egerton G, Yazdanbakhsh M, Taylor MJ. 2001. Severe reactions to filarial chemotherapy and release of Wolbachia endosymbionts into blood. Lancet 358: 1873-1875.

76. Saint André Av, Blackwell NM, Hall LR, Hoerauf A, Brattig NW, Volkmann L, et al. 2002. The role of endosymbiotic Wolbachia bacteria in the pathogenesis of river blindness. Science 295: 1892-1895. 